\title{
Corrigendum
}

\section{Corrigendum to "Structural stability and thermal expansion of TiTaNbMoZr refractory high entropy alloy" [J. Alloy. Compd. 892 (2021) 162154]}

\author{
Madhusmita Behera ${ }^{\mathrm{a}, *}$, Ajit Panigrahi $^{\mathrm{a}}$, Matthias Bönisch ${ }^{\mathrm{b}}$, Gyan Shankar ${ }^{\mathrm{c}}$, \\ Pratima Kumari Mishra ${ }^{a}$ \\ ${ }^{a}$ Advanced Materials Technology Department, CSIR-Institute of Minerals and Materials Technology, Bhubaneswar 751013, India \\ ${ }^{\mathrm{b}}$ Department of Materials Engineering, KU Leuven, Leuven 3001, Belgium \\ ${ }^{\mathrm{c}}$ Department of Materials Engineering, Indian Institute of Science, Bangalore 560012, India
}

The authors regret that the printed version of the article contained some minor errors. These errors are needed corrections. The authors would like to apologize for the inconvenience caused. These minor errors are not going to affect the results and conclusions.

The errors that are needed corrections:
1. In research highlights, 2nd bulleted highlight "B-major and Bminor phase correspond to DR and IDR, respectively" should be removed.

2. The images of Fig. 3 and Fig. 4 have been exchanged; however, the captions of Fig. 3 and Fig. 4 are correct. 